\title{
A RARE AND DISTINCT BILATERAL OVARIAN TUMOR : CARCINOSARCOMA
}

\author{
F. Limaiem ${ }^{1}$, Ahmed Halouani ${ }^{1}$, Khalil Saffar ${ }^{2}$, and saadia bouraoui ${ }^{1}$ \\ ${ }^{1}$ University Hospital Center Mongi Slim \\ ${ }^{2}$ University of Tunis El Manar
}

April 11, 2021

\begin{abstract}
Carcinosarcomas are aggressive biphasic neoplasms composed of high-grade, malignant, epithelial, and mesenchymal elements. They usually occur in the uterus and rarely involve the ovaries. Only $10 \%$ of them are bilateral. Their diagnosis relies on histological examination coupled with immunohistochemistry.
\end{abstract}

\section{INTRODUCTION}

Carcinosarcoma is a malignant mixed mesodermal neoplasm accounting for less than $1 \%$ of all ovarian tumors. It is an aggressive tumor, composed of both carcinomatous and mesenchymal components. Ovarian carcinosarcomas are about three times less prevalent than those of the uterus [1]. To the best of our knowledge, less than 400 cases of ovarian carcinosarcomas have been reported in the literature and only $10 \%$ of them are bilateral $[2,3]$. In this paper, we report a new case of bilateral ovarian carcinosarcoma in a 52-year-old woman. Our aim was to recall the clinical and pathological features of this rare and distinct neoplasm.

\section{CLINICAL HISTORY:}

A 52-year-old postmenopausal and nulliparous woman with a familial past medical history of gynecological cancer, presented with a four-month history of progressive diffuse abdominal pain, and distension. Physical examination revealed an abdominal swelling with perception of pelvic masses. Contrast-enhanced computed tomography scan of the abdomen revealed bilateral variegated ovarian masses with heterogeneous enhancement (Figure 1A). The right ovarian mass measured 43x53×60 $\mathrm{mm}$ and the left ovarian mass measured $75 \times 76 \times 89 \mathrm{~mm}$. The preoperative serum level of cancer antigen 125 (CA125) was elevated to $67 \mathrm{U} / \mathrm{ml}$ (normal range: < 35.0), whereas the carcinoembryonic antigen (CEA) and cancer antigen 19-9 (CA19-9) values were within the respective normal ranges. At laparotomy, the surgeon found bilateral ovarian masses and massive ascites. The left ovarian mass was adherent to the uterus and to the sigmoid colon. Intraoperative frozen section analysis of both ovarian masses concluded to a malignant neoplasm. The patient underwent a total abdominal hysterectomy, bilateral salpingo-oophorectomy, bilateral pelvic lymph node dissection, para-aortic lymph nodal sampling, peritoneal biopsies, and total omentectomy.

Grossly, both ovarian masses were ill defined, lobulated and friable with capsular rupture. The external surface showed numerous fragmented pieces, which were soft, encephaloid, grey, glistening with areas of hemorrhage (Figure 1B). On cut section, the tumors were solid, grey white showing variegated appearance with areas of hemorrhage and necrosis (Figure 1C). We did not notice residual ovarian tissue. Moreover, there was tumor invasion on the left side of the uterine corpus (Figure 1D). Histological examination of both ovarian masses showed a malignant biphasic tumor proliferation. The major part of the tumor was sarcomatous (Figure 2A) with the carcinomatous part well differentiated serous adenocarcinoma (Figure 
2C). Heterologous elements including cartilage (Figure 2B) and adipose tissue (Figure 2 D) were found in the tumor. Mitotic activity was frequent (8-9/10 high power field). We also noted the presence of tumor necrosis. Immunohistochemical study showed positive immunostaining of the sarcomatous component with Demin, Smooth Muscle Actin and Calponin. Cytokeratin highlighted the epithelial component. Omentum, lymph nodes and peritoneal biopsy were negative for malignant cells. Cytological examination of the ascitic fluid did not reveal the presence of malignant cells. Based on the histopathological and immunohistochemical findings, the final diagnosis was that of bilateral ovarian carcinosarcoma classified as stage IIA according to the International Federation of Gynecology and Obstetrics (FIGO) 2014. The postoperative course was uneventful. The patient was planned for adjuvant chemotherapy: six cycles of combination with paclitaxel and carboplatin. At present, she is still being followed-up.

\section{DISCUSSION}

Carcinosarcomas also called malignant mixed Mullerian tumors are highly aggressive biphasic neoplasms composed of high-grade, malignant, epithelial, and mesenchymal elements. They usually occur in the uterus and rarely involve the ovaries. Most patients with ovarian carcinosarcomas are postmenopausal of low parity and older than 60 years. Our patient was a 52-year-old, nulliparous, and postmenopausal woman. Only $10 \%$ of carcinosarcomas are bilateral as it was the case in our patient. Carcinosarcomas frequently present with widespread metastases at the time of diagnosis [4]. The most common symptoms include abdominal pain, distension, nausea, vomiting, and weight loss [5]. Our patient presented with a four-month history of abdominal pain and distension. The pathogenesis of ovarian carcinosarcoma is still debated. The collision theory stipulates that the two components of the tumor are of different origin, whereas the combination theory suggests that the two components of the tumor derive from a common epithelial stem cell [6]. Some authors have reported an association between ovarian carcinosarcoma and pelvic irradiation [7]. Approximately 74 to $90 \%$ of patients with ovarian carcinosarcoma will have an elevated CA-125 as it was the case of our patient [4]. The radiological features of ovarian carcinosarcomas are non-specific and are similar to ovarian epithelial neoplasms. In the present case, ultrasonography and contrast-enhanced computed tomography scan of the abdomen revealed bilateral variegated ovarian masses with heterogeneous enhancement. Grossly, these tumors are large (mean size, $14 \mathrm{~cm}$ ) and predominantly solid with frequent cystic degeneration and extensive hemorrhage and necrosis [8]. Histologically, carcinosarcomas are composed of high-grade carcinoma and sarcoma. One or the other may predominate. In our case, the sarcomatous component predominated. Both components are distinct but are typically intermingled with one another. The carcinomatous component is most often a high-grade serous, endometrioid, or undifferentiated adenocarcinoma and rarely clear cell adenocarcinoma or squamous cell carcinoma [8]. The sarcomatous elements are classified as homologous when the stromal component has a non-specific appearance or heterologous when rhabdomyosarcoma, chondrosarcoma (these being the most common), osteosarcoma, rarely liposarcoma or angiosarcoma are present. The presence of heterologous components can be confirmed by immunohistochemistry including desmin, myogenin and myo D1 for rhabdomyosarcoma or S100 for chondrosarcoma [8]. There is no existing consensus for the treatment of ovarian carcinosarcomas. However, the mainstay of treatment relies on optimal surgical cytoreduction followed by platinum-based chemotherapy. Combination chemotherapy with ifosfamide and cisplatin or taxol and carboplatin is favored. This results in improved progression-free survival according to large retrospective series [9]. The prognosis of ovarian carcinosarcoma is poor with a median survival reported in the majority of the studies around 11 months [10]. The adverse prognostic factors of carcinosarcomas include the presence of extra-ovarian sarcomatous elements, the advanced stage and age, the suboptimal cytoreduction, the stromal predominant tumors and tumors with serous epithelial component $[8]$.

In conclusion, ovarian carcinosarcoma is a very rare tumor with an aggressive clinical course and a poor prognosis. The definitive diagnosis of carcinosarcoma relies on the histopathological examination of the resected specimen. The immunohistochemical study highlights the biphasic components. The present case is significant owing to the extreme rarity of this ovarian neoplasm. We must record such cases for future reference with regard to the treatment provided and the outcome achieved. 


\section{CONFLICT OF INTEREST STATEMENT}

None declared.

\section{AUTHORS' CONTRIBUTIONS:}

Dr Faten LIMAIEM prepared, organized, wrote, and edited all aspects of the manuscript. She performed the gross and microscopic evaluation of the pathology specimen. She prepared all of the histology figures in the manuscript. She read, edited, and approved the final version of the manuscript.

All authors should have made substantial contributions to the manuscript.

Dr Ahmed HALOUANI and Dr Khalil SAFFAR, participated in

The conception and design of the study,

The acquisition of data, analysis and interpretation of the data,

Pr Saadia BOURAOUI participated in

The drafting of the article and revising it critically for important intellectual content,

The gross and microscopic evaluation of the pathology specimen.

All authors participated in the

Final approval of the manuscript before its submission

\section{ETHICAL APPROVAL}

All procedures performed were in accordance with the ethical standards. The examination was made in accordance with the approved principles.

\section{ACKNOWLEDGEMENT STATEMENT}

I would like to offer my special thanks to my friend Mrs Saïda Cherni who prepared the microscopic slides. Published with written consent of the patient.

\section{DATA AVAILABILITY}

In accordance with the DFG Guidelines on the Handling of Research Data, we will make all data available upon request.

\section{REFERENCES}

[1] George EM, Herzog TJ, Neugut AI, et al. Carcinosarcoma of the ovary: natural history, patterns of treatment, and outcome. Gynecol Oncol. 2013;131(1):42-5.

[2]. Harris MA, Delap LM, Sengupta PS, et al. Carcinosarcoma of the ovary. Br J Cancer 2003;88(5):654-7.

[3] Prachi S, Pankaj P, Yelikar BR. Bilateral ovarian carinosarcoma-a rare entity. Int J Med Res Health Sci. 2015;4(1):242-4.

[4] Brown E, Stewart M, Rye T, et al. Carcinosarcoma of the ovary: 19 years of prospective data from a single center. Cancer 2004; 100:2148-53.

[5] Lamb MR, Gertsen E, Middlemas E. Carcinosarcoma of the ovary: Case report and literature review. Tenn Med 2012; 105:41-2.

[6] Petru E, Haas J, Beganovic S, et al. Carcinosarcoma (Malignant Mixed Muellerian Tumor) of the Ovary-A Single Institution Experience of 25 years. Int J Gynecol Clin Pract 2015,2:107.

[7] Wei LH, Huang C Y, Cheng SP, et al. Carcinosarcoma of ovary associated with previous radiotherapy. Int J Gynecol Cancer 2001; 11:81-4. 
[8] Kunkel J, Peng Y, Tao Y, et al. Presence of a sarcomatous component outside the ovary is an adverse prognostic factor for primary ovarian malignant mixed mesodermal/mullerian tumors: a clinicopathologic study of 47 cases. Am J Surg Pathol 2012; 36: 831-7.

[9] Leiser AL, Chi DS, Ishill NM, et al. Carcinosarcoma of the ovary treated with platinum and taxane: the memorial SloanKettering Cancer Center experience. Gynecol Oncol 2007;105: 657-61.

[10] Rauh-Hain JA, Growdon WB, Rodriguez N, et al. Carcinosarcoma of the ovary: a case-control study. Gynecol Oncol 2011;121: 477-81.

\section{FIGURE LEGENDS}

\section{Multipanel Figure 1:}

Figure 1 A: Contrast enhanced CT scan axial image showing bilateral variegated ovarian masses with heterogeneous enhancement.

Figure 1 B: Grossly, both ovarian masses were ill defined, lobulated and friable with capsular rupture. The external surface showed numerous fragmented pieces, which were soft, encephaloid, grey, glistening with areas of hemorrhage.

Figures 1 C: On cut section of the resected ovarian mass, the tumor was friable, whitish with foci of hemorrhage.

Figures 1 D: Total hysterectomy specimen showing tumor invasion of the uterine corpus (black arrow).

Multipanel Figure 2:

Figure 2 A: Microscopically the ovarian tumor shows the presence of both epithelial (arrow) and stromal components, ( Hematoxylin and eosin, magnification x 40).

Figure 2 B: Heterologous elements including neoplastic cartilage (arrows) within a sarcomatous component, ( Hematoxylin and eosin, magnification x 40).

Figure 2 C: Epithelial component of the tumor (endometrioid carcinoma): glandular structures within a sarcomatous background. (Hematoxylin and eosin, magnification x 400).

Figure 2 D: Heterologous elements including lipoblasts within the tumor proliferation, (Hematoxylin and eosin, magnification x 400). 

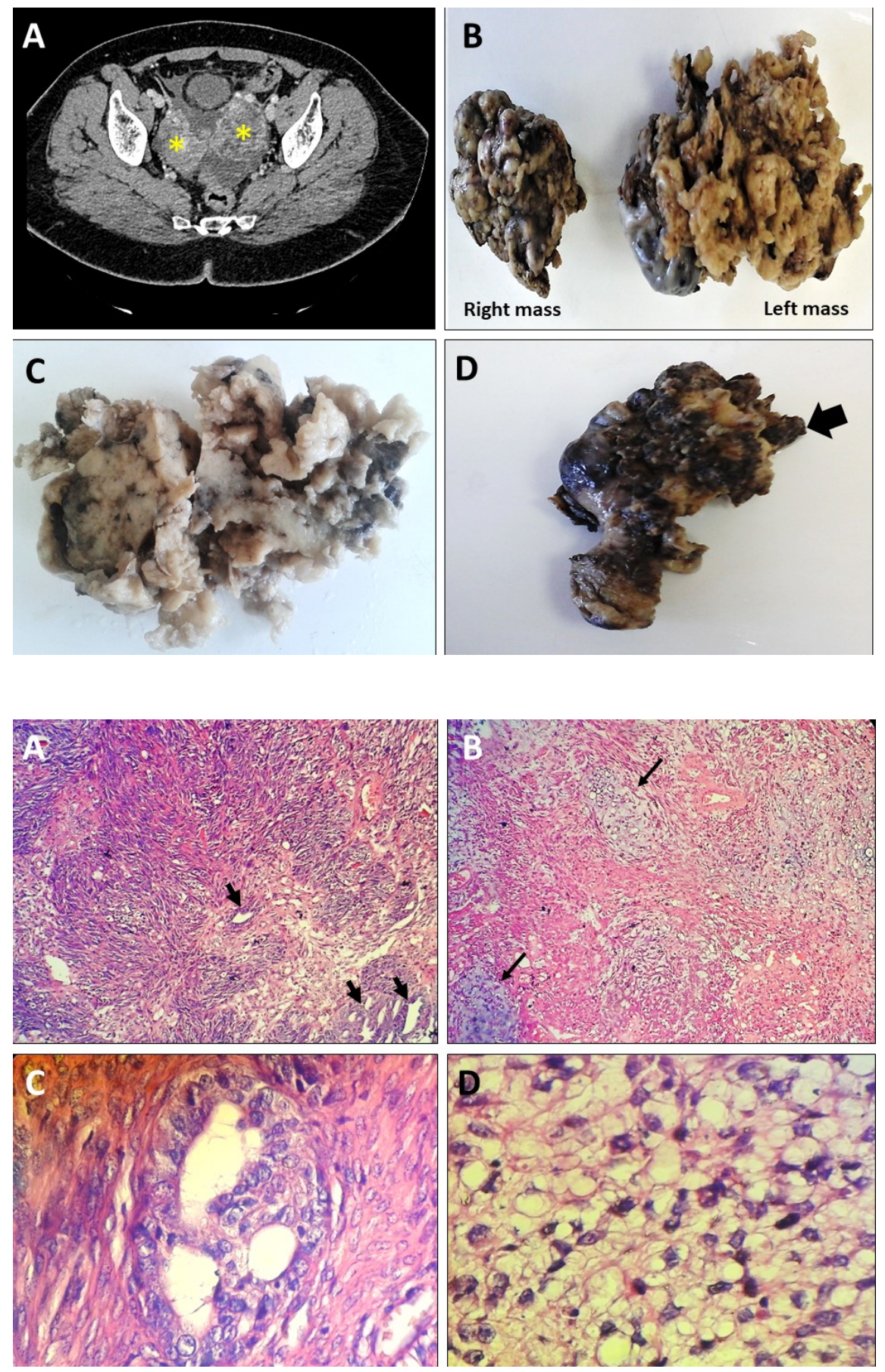